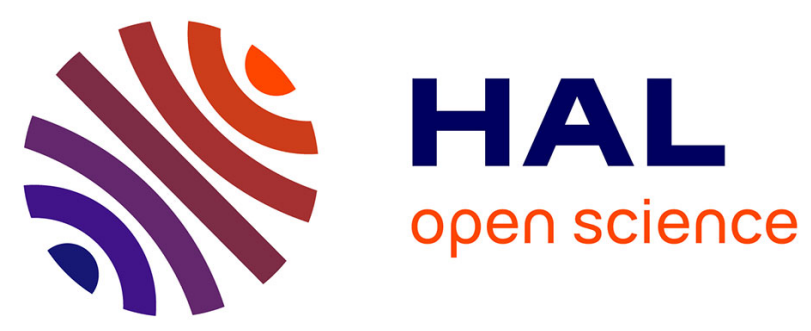

\title{
Conditions for the sliding-bouncing transition for the interaction of a bubble with an inclined wall
}

\author{
Christophe Barbosa, Dominique Legendre, Roberto Zenit
}

\section{To cite this version:}

Christophe Barbosa, Dominique Legendre, Roberto Zenit. Conditions for the sliding-bouncing transition for the interaction of a bubble with an inclined wall. Physical Review Fluids, 2016, vol. 1 ( $\mathrm{n}^{\circ}$ 3), pp. 32201/1-32201/10. 10.1103/PhysRevFluids.1.032201 . hal-01792573

\section{HAL Id: hal-01792573 \\ https://hal.science/hal-01792573}

Submitted on 15 May 2018

HAL is a multi-disciplinary open access archive for the deposit and dissemination of scientific research documents, whether they are published or not. The documents may come from teaching and research institutions in France or abroad, or from public or private research centers.
L'archive ouverte pluridisciplinaire HAL, est destinée au dépôt et à la diffusion de documents scientifiques de niveau recherche, publiés ou non, émanant des établissements d'enseignement et de recherche français ou étrangers, des laboratoires publics ou privés. 


\section{Open Archive TOULOUSE Archive Ouverte (OATAO)}

OATAO is an open access repository that collects the work of Toulouse researchers and makes it freely available over the web where possible.

This is an author-deposited version published in: http://oatao.univ-toulouse.fr/ Eprints ID: 19936

To link to this article: DOI: 10.1103/PhysRevFluids.1.032201 URL: http://dx.doi.org/10.1103/PhysRevFluids.1.032201

To cite this version: Barbosa, Christophe and Legendre, Dominique and Zenit, Roberto Conditions for the sliding-bouncing transition for the interaction of a bubble with an inclined wall. (2016) Physical Review Fluids, vol. 1 (n ${ }^{\circ}$ 3). pp. 32201/1-32201/10. ISSN 2469$990 \mathrm{X}$

Any correspondence concerning this service should be sent to the repository administrator: staff-oatao@ listes-diff.inp-toulouse.fr 


\title{
Conditions for the sliding-bouncing transition for the interaction of a bubble with an inclined wall
}

\author{
C. Barbosa, ${ }^{1,2}$ D. Legendre, ${ }^{2, *}$ and R. Zenit ${ }^{1, \dagger}$ \\ ${ }^{1}$ Instituto de Investigaciones en Materiales, Universidad Nacional Autónoma de México, \\ Apartado Postal 70-360, México Distrito Federal 04510, Mexico \\ ${ }^{2}$ Institut de Mécanique des Fluides de Toulouse, 1 Allée du Professeur Camille Soula, 31400 Toulouse, France \\ (Received 30 March 2016; published 12 July 2016)
}

\begin{abstract}
In this study we analyze the interaction of a single rising bubble with an inclined wall. We conduct experiments considering different liquids and bubble sizes, to cover a wide range of Reynolds and Weber numbers, with wall angles from nearly horizontal to nearly vertical. For all cases, the bubble initially collides with the wall; after the initial interaction, in accord with previous studies, the bubble either steadily slides on the wall or ascends, colliding repeatedly with it. Considering a force balance for the bubble motion on the wall, we propose a set of conditions for the transition from sliding to bouncing that is validated with the present and previous data.
\end{abstract}

DOI: 10.1103/PhysRevFluids.1.032201

\section{INTRODUCTION}

The study of two-phase bubbly flows is largely justified by the numerous engineering applications and natural phenomena in which such flows occur. Significant advances have been reached in recent years due to the mature understanding of the hydrodynamic forces that affect the motion of single bubbles ascending in Newtonian liquids [1]. There is a clear understanding of the effects of viscous and inertial forces around interacting bubbles [2]. Conversely, despite their omnipresence, the understanding of wall effects is still modest.

The interaction of ascending bubbles with vertical walls has been studied by several authors [3-6]. Similar to the case of bubble pair interactions [7], the force between the bubble and the wall transitions from repulsive to attractive as the Reynolds number increases [3]. When the wall attracts the bubble, it is possible to observe repeated bouncing [4,5]. According to De Vries et al. [4], the bounce and rebound are affected by the wake behind the bubble. The process of bubble bouncing against a horizontal wall was analyzed in detail by Zenit and Legendre [8]; they identified the conditions for rebound, as opposed to arrest, considering the dependence of the coefficient of restitution with the Stokes and capillary numbers. For the interaction of a bubble with an inclined wall, an interesting phenomenon occurs: The motion of the bubble can be either repeated bouncing (as for a vertical wall) or steady sliding. The same bubble-fluid combination can exhibit the two behaviors; the type of motion observed is determined by the inclination of the wall. Tsao and Koch [9] originally reported this phenomenon. They proposed that the transition occurred for a single value of the Weber number, but did not fully explain the physical process for the transition.

The sliding motion of bubbles on inclined walls has been studied to some extent. Aussillous and Quéré [10] proposed scalings for the wall velocity, considering different regimes of bubble deformation; however, they restricted their analysis for nearly horizontal walls. The bouncing motion of bubbles on a wall has been addressed mainly for the case when the wall is vertical. De Vries et al. [4] conducted flow visualization of the process and concluded that the bubble wake significantly influences the rebound off the wall.

\footnotetext{
*legendre@imft.fr

†zenit@unam.mx
} 


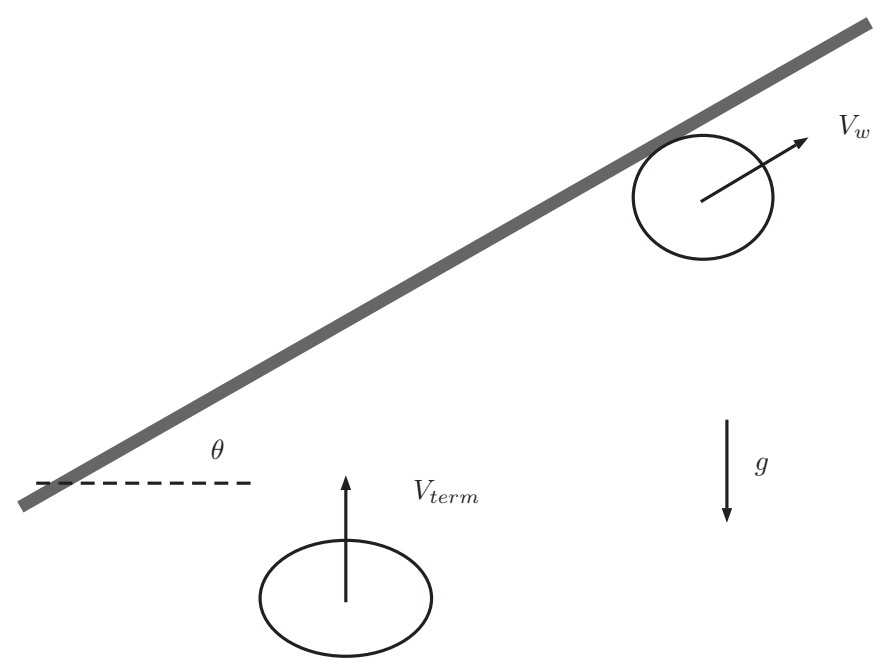

FIG. 1. A bubble rises in a viscous liquid at terminal conditions $V_{\text {term }}$ and $\chi_{\text {term }}$; it then collides with a wall, inclined at an angle $\theta$. After a transient phase, the bubble reaches a new time-average steady velocity $V_{w}$.

In the present study we investigate experimentally the motion of high Reynolds number and moderate Weber number bubbles interacting with an inclined wall, closely following the original investigation by Tsao and Koch [9]. Unlike them, we substantially extend the range of parameters by considering different fluids (with different viscosities, densities, and surface tensions) and bubble sizes. Based on this wider perspective, we are able better characterize the phenomenon and explain the physical mechanism that leads to the transition from sliding to bouncing.

\section{EXPERIMENTAL SETUP}

The experimental arrangement used in this investigation is shown in Fig. 1. Single air bubbles were generated from the bottom of a tank, filled with a viscous fluid, using a capillary tube and a syringe pump. Before reaching the wall, the bubble attains its terminal state, reaching a terminal velocity $V_{\text {term }}$ and shape $\chi_{\text {term }}=d_{\mathrm{ma}} / d_{\mathrm{mi}}$, where $d_{\mathrm{ma}}$ and $d_{\mathrm{mi}}$ are the major and minor bubble axes, respectively.

The height, width, and length of the rectangular glass tank were, respectively, 50, 10, and $40 \mathrm{~cm}$. Within the tank, a glass plate upon which the rising bubble will bounce was used as the test wall. The inclination angle of the wall was varied from $5^{\circ}$ to $80^{\circ}$, with an accuracy of $\pm 0.1^{\circ}$. To obtain a wide range of experimental conditions, six different liquids and three capillary sizes were used. The properties and values of the dimensionless numbers for each liquid are shown in Table I. The viscosity and the surface tension were measured with a stress controlled (MCR101) Rheometer and a Wilhelmy balance with a DuNouy ring, respectively.

The bubble-wall interaction was recorded with a high-speed camera (Phantom v9.1, $1632 \times 1200$ pixels) at a rate of at least 1000 frames/s with a 120-mm Nikkon lens. Considering the optical arrangement (lens, camera and working distance) a spatial resolution of $11.5 \mu \mathrm{m} /$ pixel was obtained. The array was illuminated with a light-emitting diode panel from the back. Each experiment was repeated at least 5 times to ensure statistical significance.

The bubble location and the wall position were determined for each frame using MATLAB. From the bubble image, the equivalent diameter was calculated as $D_{\mathrm{eq}}=\left(d_{\mathrm{ma}}^{2} d_{\mathrm{mi}}\right)^{1 / 3}$. The instantaneous bubble velocity was calculated using the evolution of position in time, considering a central difference scheme. We characterize each experiment (a combination of a bubble size in a liquid) 
TABLE I. Physical properties for all the experiments conducted in this investigation. In all cases, the liquids were mixtures of water (W), glycerol $(\mathrm{G})$, and tri-ethanol amine $(\mathrm{T})$; percentages in the second column are by weight. Three experiments were performed using silicon oil (SO). The type of trajectory for bubbles before reaching the wall is shown on the first column: rectilinear $(\mathrm{R})$ or oscillatory $(\mathrm{O})$.

\begin{tabular}{|c|c|c|c|c|c|c|c|c|c|}
\hline Experiment & $\begin{array}{c}\text { Composition } \\
(\%)\end{array}$ & $\begin{array}{c}\rho \\
\left(\mathrm{kg} / \mathrm{m}^{3}\right)\end{array}$ & $\begin{array}{c}\mu \\
(\mathrm{mPas})\end{array}$ & $\begin{array}{c}\sigma \\
(\mathrm{mN} / \mathrm{m})\end{array}$ & $\begin{array}{c}D_{\mathrm{eq}} \\
(\mathrm{mm})\end{array}$ & $\mathrm{Re}_{\text {term }}$ & $\mathrm{We}_{\text {term }}$ & $\chi_{\text {term }}$ & $\theta_{\text {trans }}$ \\
\hline E1, $(\mathrm{R})$ & SO 100 & 855 & 1.280 & 18.0 & 1.1 & $138 \pm 3$ & $1.8 \pm 0.05$ & $1.31 \pm 0.02$ & $80^{\circ}$ \\
\hline $\mathrm{E} 2, \diamond(\mathrm{R})$ & SO 100 & 855 & 1.280 & 18.0 & 1.2 & $172 \pm 8$ & $2.6 \pm 0.17$ & $1.32 \pm 0.05$ & $80^{\circ}$ \\
\hline $\mathrm{E} 3, \bullet(\mathrm{O})$ & SO 100 & 855 & 1.280 & 18.0 & 2.2 & $313 \pm 7$ & $4.7 \pm 0.23$ & $2.06 \pm 0.04$ & $45^{\circ}$ \\
\hline $\mathrm{E} 4, \boldsymbol{( R )}$ & W-G 80-20 & 1045 & 1.555 & 70.2 & 1.7 & $305 \pm 7$ & $1.9 \pm 0.09$ & $1.26 \pm 0.04$ & $70^{\circ}$ \\
\hline $\mathrm{E} 5, \triangleleft(\mathrm{O})$ & W-T 99.88-0.12 & 1001 & 1.529 & 61.3 & 2.8 & $250 \pm 9$ & $2.0 \pm 0.11$ & $1.16 \pm 0.02$ & $70^{\circ}$ \\
\hline $\mathrm{E} 6, \Delta(\mathrm{O})$ & W-G 85-15 & 1033 & 1.363 & 70.0 & 1.6 & $367 \pm 16$ & $2.1 \pm 0.16$ & $1.44 \pm 0.05$ & $65^{\circ}$ \\
\hline $\mathrm{E} 7, \nabla(\mathrm{O})$ & W-G 90-10 & 1021 & 1.165 & 70.6 & 1.6 & $469 \pm 14$ & $2.5 \pm 0.15$ & $1.63 \pm 0.08$ & $60^{\circ}$ \\
\hline $\mathrm{E} 8, \square(\mathrm{O})$ & W-G 95-5 & 1009 & 1.038 & 70.8 & 1.7 & $536 \pm 21$ & $2.6 \pm 0.20$ & $1.58 \pm 0.07$ & $60^{\circ}$ \\
\hline E9, $(\mathrm{O})$ & W-G 90-10 & 1021 & 1.165 & 70.6 & 2.9 & $640 \pm 77$ & $2.6 \pm 0.5$ & $1.69 \pm 0.28$ & $50^{\circ}$ \\
\hline $\mathrm{E} 10, \bullet(\mathrm{O})$ & W 100 & 998 & 0.955 & 72.6 & 1.6 & $601 \pm 40$ & $2.7 \pm 0.3$ & $1.78 \pm 0.10$ & $50^{\circ}$ \\
\hline $\mathrm{E} 11, \star(\mathrm{O})$ & W 100 & 998 & 0.955 & 72.6 & 3.1 & $955 \pm 46$ & $3.6 \pm 0.3$ & $1.93 \pm 0.13$ & $45^{\circ}$ \\
\hline
\end{tabular}

with the terminal Reynolds and Weber numbers $\operatorname{Re}_{\text {term }}=\rho V_{\text {term }} D_{\text {eq }} / \mu$ and $\mathrm{We}_{\text {term }}=\mu V_{\text {term }}^{2} D_{\text {eq }} / \sigma$, respectively, where $V_{\text {term }}$ is the terminal bubble velocity.

After the bubble reaches the wall and collides with it, the motion reaches a new time-average steady state. In short, it could slide at constant speed or repeatedly bounce with a constant mean velocity. We characterize this final state considering also the Reynolds and Weber numbers but using the wall velocity $V_{w}$ such that $\operatorname{Re}_{w}=\operatorname{Re}_{\text {term }} V_{w} / V_{\text {term }}$ and $\mathrm{We}_{w}=\operatorname{We}_{\text {term }}\left(V_{w} / V_{\text {term }}\right)^{2}$. The ratio $V_{w} / V_{\text {term }}$ is determined experimentally. Its value depends on the inclination angle $\theta$, but is also influenced by the fluid properties and possibly the contamination level of the bubble surface.

Figure 2(a) shows a $\mathrm{Re}_{\text {term }}-\mathrm{We}_{\text {term }}$ map for all the experiments conducted in this investigation. For each experiment, there is a certain variability of $\mathrm{Re}_{\text {term }}$ and $\mathrm{We}_{\text {term }}$ (see Table I), which indicates small variations of the bubble size and terminal velocity for the same nominal conditions. The terminal Reynolds extends approximately from 100 to 1000, while the terminal Weber spans roughly from 1.5 to 5 . The bubble aspect ratio varies from 1.2 to 2.1 . For this range of values, the bubble are spheroidal and ascend in an either rectilinear or zigzag manner. In Fig. 2(b) the Reynolds number is shown as a function of the wall angle, which extends from nearly horizontal to nearly vertical. In the figure, the experiments that exhibit sliding (bouncing) are shown with closed (open) symbols.

A few visualization experiments were conducted using the particle image velocimetry technique. The details of this setup can be found in Ref. [11]. The flow was illuminated with a laser sheet (Nd:YLF Litron laser, $527 \mathrm{~nm}, 10 \mathrm{~mJ}, 500 \mathrm{~Hz}$ ) and neutrally buoyant silver-coated glass spheres $(10 \mu \mathrm{m}$ in diameter) were added as particle tracers. The images were processed with the DYNAMICSTUDIO software (Dantec Dynamics), using an adaptive-correlation technique with interrogation areas of $16 \times 16$ pixels with a $75 \%$ overlap. 


\section{BARBOSA, D. LEGENDRE, AND R. ZENIT}

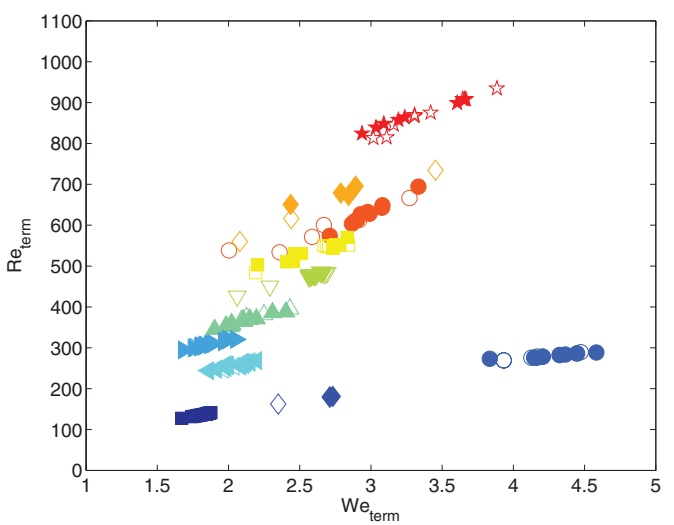

(a)

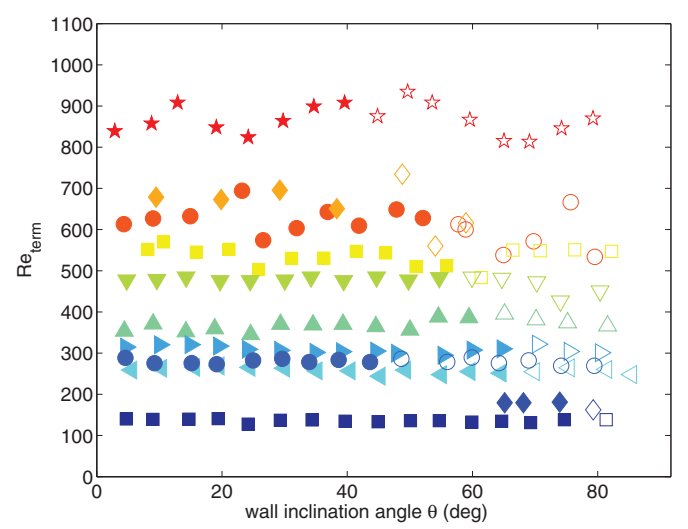

(b)

FIG. 2. (a) Map of terminal Reynolds $\mathrm{Re}_{\text {term }}$ and Weber $\mathrm{We}_{\text {term }}$ numbers showing all the experiments conducted in this investigation and (b) terminal Reynolds number $\mathrm{Re}_{\text {term }}$ as a function of wall inclination angle $\theta$. The symbols are according to Table I. In all cases, the closed and open symbols show the experiments in which sliding or bouncing was observed, respectively.

\section{RESULTS}

Two types of bubble-wall interaction

In agreement with what was previously reported in Ref. [9], after the initial interaction, the motion of the bubble transitions from steady sliding to repeated bouncing when the wall inclination angle surpasses a certain critical value. The two types of bubble-wall interaction are shown in Figs. 3(a)

\section{$100 \%$}

(a)

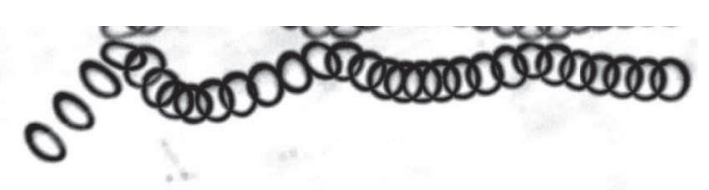

(b)

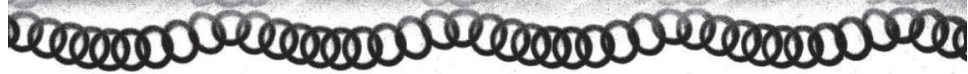

(c)

FIG. 3. Bubble motion for two typical behaviors: (a) impact and sliding for $\theta=50^{\circ}$ and (b) impact and bouncing for $\theta=60^{\circ}$. In both cases $D_{\text {eq }}=1.6 \mathrm{~mm}, \mathrm{Re}_{\text {term }}=500$, and $\mathrm{We}_{\text {term }}=2.3$, corresponding to experiment E7 from Table I. The image is composed by superposing bubble positions at different instants, $\Delta t=5 \mathrm{~ms}$. Note that the image was rotated to make the wall appear horizontal, in both cases. (c) Repeated bouncing behavior for $\theta=60^{\circ}$; the experimental conditions are the same as in (b) but further along the plate. 
CONDITIONS FOR THE SLIDING-BOUNCING ...
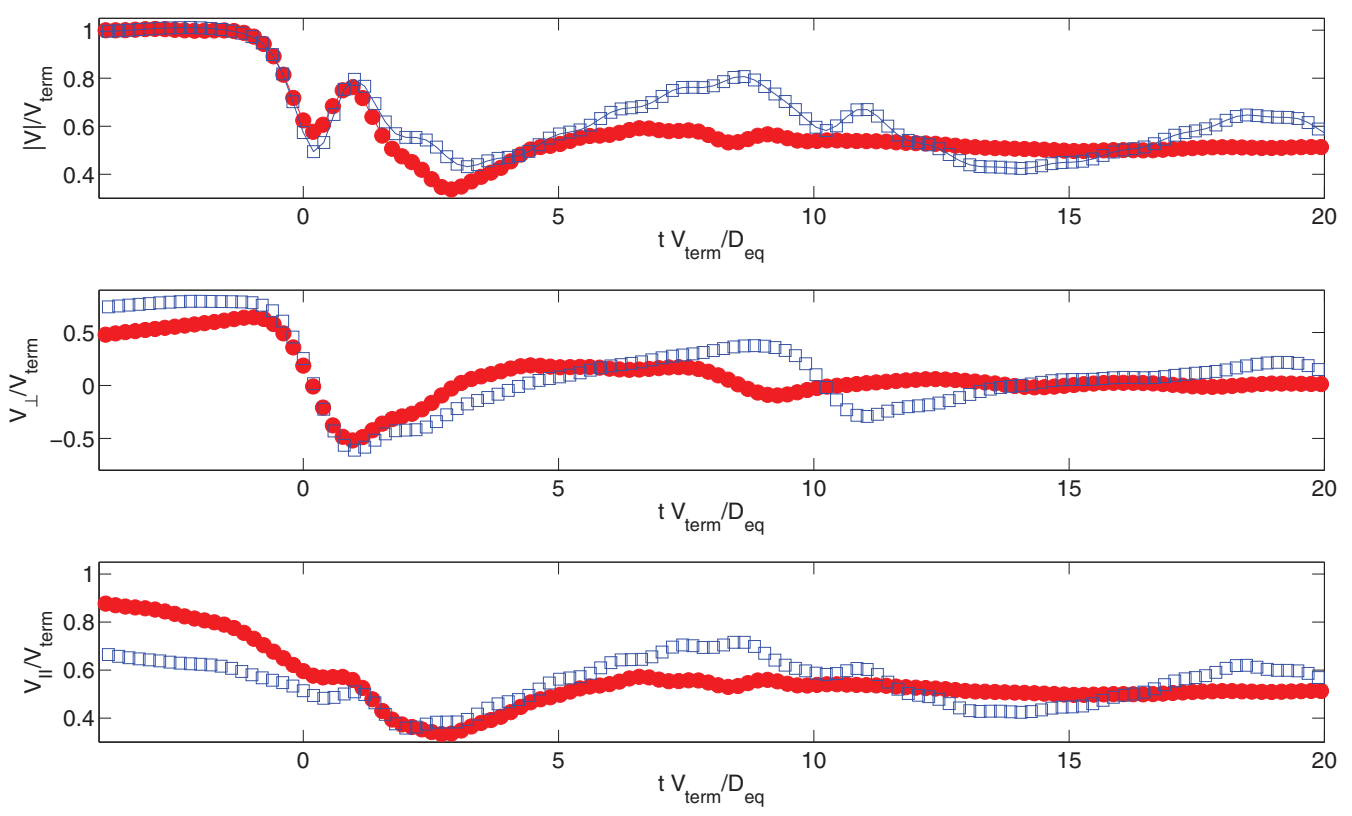

FIG. 4. Evolution of the normalized bubble velocity as a function of normalized time $t D_{\text {eq }} / V_{\text {term }}$ for the experiments shown as closed and open symbols. The top, middle, and bottom plots show the velocity magnitude $|V| / V_{\text {term }}$, the perpendicular velocity $V_{\perp} / V_{\text {term }}$, and the parallel velocity $V_{\|} / V_{\text {term }}$, respectively.

and 3(b) for an ellipsoidal bubble (corresponding to experiment E3 from Table I) for two inclination angles of $50^{\circ}$ and $60^{\circ}$, respectively.

For the cases shown in the figure, for both angles, after a transient phase during which the bubble touches and bounces off the wall, a wall-terminal state is reached. For small inclination angles, the bubble steadily slides against the wall with a constant velocity; for inclination angles higher than the critical value, the bubble bounces repeatedly in time with a constant amplitude and period. This same behavior was observed for all the experiments conducted in this investigation. For each case, the transition angle was determined. Note that, in their experiments, Tsao and Koch [9] only considered water and two bubble diameters; hence, the extent of experimental parameters was narrow. The distinction between the two regimes can also be observed by plotting the evolution of the bubble velocity in time, as shown in Fig. 4. In the plot three measures of the bubble velocity are shown as a function of time: the magnitude of the bubble velocity $|V|$, the component of velocity perpendicular to the wall $V_{\perp}$, and the tangential bubble velocity $V_{\|}$. The time $t=0$ is defined as the moment at which the bubble normal velocity vanishes. Clearly, the collision process is complex; the kinetic energy associated with the bubble motion is transformed into deformation energy or is dissipated by viscous effects, as discussed in Ref. [8]. For this case, since the wall is inclined, the motion and rebound also are important components in the direction parallel to the wall. Once this transient process is completed, for about $t D_{\text {eq }} / V_{\text {term }}>10$, the bubble motion reaches a new time-average steady state.

An additional set of tests was conducted for experiment E10 (see Table I), in which the bubbles were released just beneath the wall. In this case, the bubbles did not attain their terminal state before the initial interaction with the wall. We observed the same two regimes: sliding and bouncing. More importantly, we found that the value of the critical transition angle was not affected. Therefore, we can argue that the initial condition of the interaction does not affect the final state of motion of the bubble. 


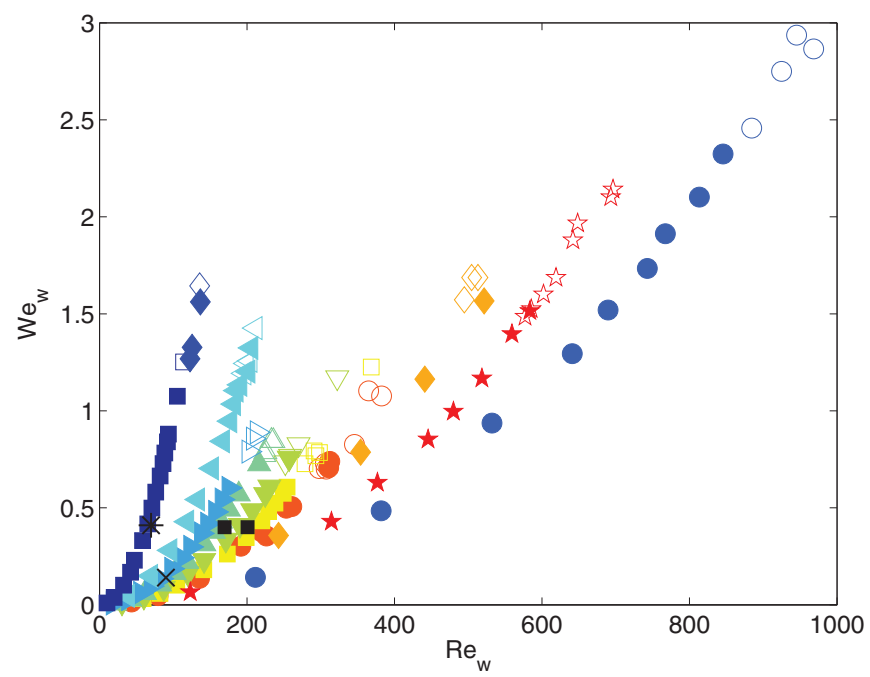

FIG. 5. Map of wall Reynolds $\mathrm{Re}_{w}$ and Weber $\mathrm{We}_{w}$ numbers showing all the experiments conducted in this investigation. The symbols are according to Table I. In all cases, the closed and open symbols show the experiments in which sliding or bouncing was observed, respectively. The black squares show the data from Tsao and Koch [9]; the asterisks and crosses are results from Takemura and Magnaudet [3] and De Vries et al. [4], respectively.

\section{PHYSICAL CONDITIONS FOR THE TRANSITION FROM SLIDING TO COLLISIONAL MODES}

Clearly, from the data shown in Table I and in Fig. 2, the critical angle for the transition varies for each liquid-bubble combination. The angles do not seem to correlate with either $\mathrm{Re}_{\text {term }}$ or $\mathrm{We}_{\text {term }}$. Tsao and Koch [9] reported that the transition occurred for $\mathrm{We}_{w}=0.4$, which implies that the transition is a result of the deformability of the bubble. Figure 5 shows a map of $\operatorname{Re}_{w}$ as a function of $\mathrm{We}_{w}$ for all the experiments conducted in this investigation. Clearly, a single value of either $\operatorname{Re}_{w}$ or $\mathrm{We}_{w}$ that characterizes the transition for all cases cannot be identified. Also from Table I and Fig. 2, the transition does not occur for a single value of $\theta_{\text {trans }}$.

Note that it is possible to also include the data corresponding to the transition to the bouncing regime for vertical walls. Takemura and Magnaudet [3] argued that the transition from wall repulsion to wall attraction was associated with the prevalence of inertial over viscous effects; hence, for such a case the transition would be characterized by a critical value of Re rather than We. The critical conditions from $[3,4]$ are also shown in the figure.

\section{Analysis}

To understand the conditions for transition we consider a simple force balance for the bubble motion in both the parallel and perpendicular directions. From above, we recognize that both $\operatorname{Re}_{w}$ and $\mathrm{We}_{w}$ are important parameters to characterize the transition. In particular, the value of $\mathrm{We}_{w}$ will determine the shape of the bubbles during their interaction with the wall [12].

To evaluate the influence of $\mathrm{We}_{w}$ on the bubble shape, we shown typical bubble shapes for the sliding regime, at angles slightly below $\theta_{\text {trans }}$. Figure 6 shows snapshots of four experiments. In Figs. 6(a) and 6(b) the bubbles have $\mathrm{We}_{w}<1$.1, for which the shape is close to spherical and the elongation is parallel to the wall, resulting from gravity effects. The images in Figs. 6(c) and 6(d), for which $\mathrm{We}_{w}>1.5$, show bubbles that are largely deformed. It is curious to observe that, for this case, the bubbles are elongated in the direction perpendicular to the wall, arguably resulting from inertial effects. 
CONDITIONS FOR THE SLIDING-BOUNCING ...

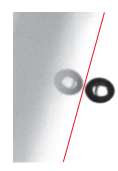

(a)E1,

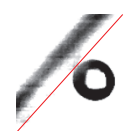

(b)E10, •

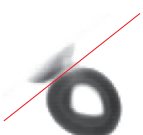

(c)E9, $>$

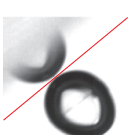

(d)E11, $\star$

FIG. 6. Images for all bubbles in the sliding motion, at an angle just below the transition. The symbols are according to Table I. (a) $\mathrm{E} 1, \mathrm{Re}_{w}=106, \mathrm{We}_{w}=1.07$, and $\theta=75^{\circ}$; (b) $\mathrm{E} 10, \mathrm{Re}_{w}=311, \mathrm{We}_{w}=0.71$, and $\theta=50^{\circ}$; (c) $\mathrm{E} 9, \operatorname{Re}_{w}=522, \mathrm{We}_{w}=1.57$, and $\theta=40^{\circ}$; and (d) $\mathrm{E} 11, \operatorname{Re}_{w}=627$, $\mathrm{We}_{w}=1.63$, and $\theta=40^{\circ}$. Images are shown on the same scale.

Let us now consider a force balance for a bubble in both the parallel and perpendicular directions. We first recognize that there is a gravitational force pushing the bubble to remain in contact with the wall. Second, we consider that there are viscous and/or inertial drag forces in the parallel wall direction. More importantly, we also argue that there is an inertial wall-repulsive force acting in the normal direction that results from the interaction of the bubble wake with the wall similar to what was previously discussed by De Vries et al. [4]. By conducting a Schlieren-type visualization, these authors were able to observe the interaction between the bubble and vortex blobs from the wake. They argued that the interaction gave rise to a lift-type force that depended on the strength of the circulation of the vortex filament. They proposed that such force was proportional to $\rho V_{w}^{2} D_{\mathrm{eq}}^{2}$. In our case, the force pushing the bubble away from the wall also results from both the vorticity in the wake (proportional to $V_{w} / D_{\text {eq }}$ ) and the proximity of the wall. The wall provides the symmetry-breaking mechanism and the vorticity interaction is known to result in a repulsive effect [7]. Since we argue that the force arises from the wake, we conjecture that it also scales with $\rho V_{w}^{2} D_{\mathrm{eq}}^{2}$.

Hence, balancing gravity with an inertial wake-induced wall force for the perpendicular direction, the criterion for bubble departure from the wall would be

$$
\rho V_{w}^{2} D_{\text {eq }}^{2} \gtrsim \rho D_{\text {eq }}^{3} g \cos \theta .
$$

For the motion in the direction parallel to the wall, we can balance the buoyancy with a viscous drag force:

$$
\mu D_{\mathrm{eq}} V_{w} \sim \rho D_{\mathrm{eq}}^{3} g \sin \theta .
$$

Taking the ratio of Eqs. (1) and (2), we have

$$
\operatorname{Re}_{w} \gtrsim \cot \theta .
$$

Therefore, we can say that when the wall Reynolds-number angle surpasses a certain critical value, the bubble will not be able to remain sliding on the wall beyond the angle $\theta$. This critical condition is expected to apply for a spheroidal bubble for which the drag force is dominated by the viscous effect [13]. In Fig. 7 we replot our results, separating the data into two different sets. In Fig. 7(a) the results corresponding to $\mathrm{We}<1.2$ are shown in terms of $\operatorname{Re}_{w}$ as a function of $\cot \theta$ as suggested by Eq. (3). The data clearly show that there is indeed a linear relation between $\operatorname{Re}_{w}$ and $\cot \theta$ for the critical conditions. For the transition, our data can be closely fitted to

$$
\operatorname{Re}_{w}=\operatorname{Re}_{0}+310 \cot \theta
$$

where $\mathrm{Re}_{0} \approx 80$. In fact, the value of $\mathrm{Re}_{0}$ coincides with the transition data for vertical walls from De Vries et al. [4] and Takemura and Magnaudet [3]. The transition conditions reported by Tsao and Koch [9] (black squares) are also in consistent agreement with Eq. (4).

For bubbles with large values of $\mathrm{We}_{w}$ (here $\mathrm{We}_{w}>1.5$ ) we observed that the transition is not given by Eq. (3). For such cases the bubbles are more deformed, as shown in Figs. 6(c) and 6(d). We conducted visualization experiments of the wake structure for this case. Figure 8 shows the flow 


\section{BARBOSA, D. LEGENDRE, AND R. ZENIT}

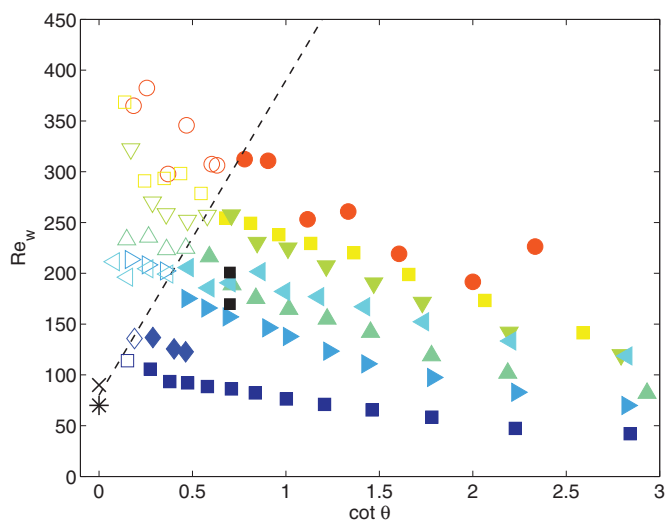

(a)

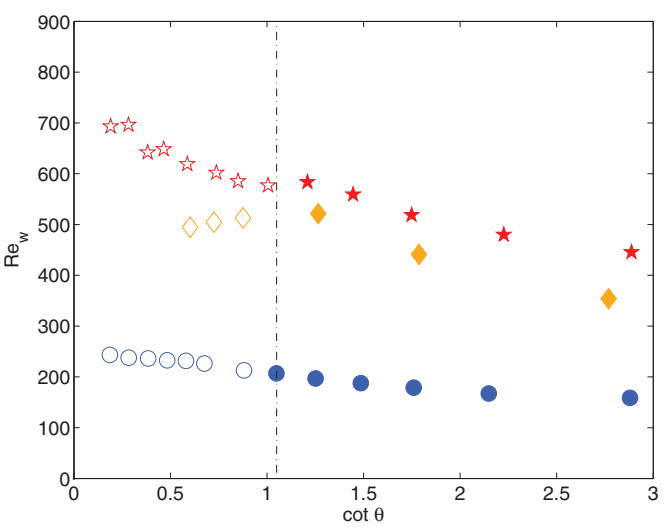

(b)

FIG. 7. Conditions for transition: (a) $\mathrm{We}_{\text {wall }}<1.2$ and wall Reynolds $\operatorname{Re}_{\text {wall }}$ as a function of $\cot \theta$ and (b) $\mathrm{We}_{\text {wall }}>1.5$ and wall Weber $\mathrm{We}_{\text {wall }}$ as a function of inclination angle $\theta$. The symbols are according to Table I. In all cases, the closed and open symbols show the experiments in which sliding or bouncing was observed, respectively. The black squares are the data from Tsao and Koch [9]; the asterisks and crosses are results from Takemura and Magnaudet [3] and De Vries et al. [4], respectively. The dashed line in (a) corresponds to Eq. (4). The vertical dash-dotted line in (b) corresponds to $\theta=43.7^{\circ}$.

around a sliding bubble for experiment E10 (see Table I) for an angle slightly below the critical one $\left(\theta=50^{\circ}\right)$. Significant vortex shedding in the bubble wake is clearly observed; hence, in this case, the resulting drag would be dominated by inertial effects.

Therefore, the force balance parallel to the wall is now

$$
\rho V_{w}^{2} D_{\text {eq }}^{2} \sim \rho D_{\text {eq }}^{3} g \sin \theta
$$

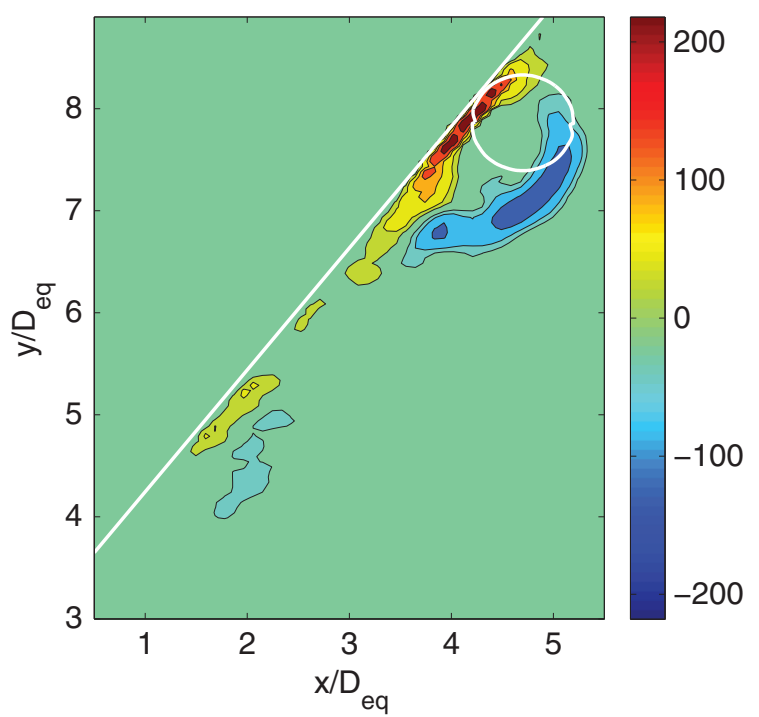

FIG. 8. Visualization of the bubble wake for a sliding bubble, slightly below the transition angle. The image was taken at steady sliding $t D_{\text {eq }} / V_{\text {term }}=54$ for E10 in Table I for $\theta=50^{\circ}$. The colors show the value of the vorticity, normalized by $D_{\text {eq }} / V_{\text {term }}$. 
The ratio of Eqs. (1) and (5) suggests that for the transition $\cot \theta$ should be constant. Figure 7(b) shows again $\operatorname{Re}_{w}$ as a function of $\cot \theta$, but only for experiments with $\mathrm{We}_{w}>1.5$. The transition is now clearly observed for a constant angle around $\cot \theta \approx 1$, corresponding to a value of $\theta \approx \pi / 4$. These data cover a significant range of $\operatorname{Re}_{w}$ (from 200 to 600).

It is important to note that the strength of the wake-induced lift and the drag force, and as a result the induced transition criteria, is probably affected by the Reynolds and Weber number corrections. Indeed, there are several factors that may affect the magnitude of these forces: the structure of the far wake, the vorticity production on the bubble surface, the bubble shape, the interaction with the wall, and even the possible presence of surfactants. The fact that our model prediction agrees well with the experiments indicates that such a dependence of the forces on Re and/or We is not significant for the range of parameters explored here.

\section{CONCLUSION}

In this investigation we studied the interaction of an air bubble rising in a viscous fluid during its interaction with an inclined wall. This particular problem had been addressed previously. Tsao and Koch [14] first reported that, for air bubbles in water, the interaction changes from steady sliding to repeated bouncing at a certain angle. They argued that the condition for transition occurred at a certain critical Weber number, based on the sliding velocity $\mathrm{We}_{w}$. Conversely, Takemura and Magnaudet [3] claimed that, for a vertical wall, the transition would occur for a certain critical value of $\mathrm{Re}_{w}$. In this investigation, due to a wider range of experimental conditions, we demonstrated that, instead, the transition results from the appearance of a wake-induced lift. Furthermore, we found that there are two different regimes for the transition depending on the value of the Weber number (based on the wall velocity), which in turn determines the shape of the bubble, the wake structure, and therefore the nature of the drag force (viscous or inertial) on it.

\section{ACKNOWLEDGMENTS}

C.B. is grateful to the CONACyT, Mexico scholarship program for their financial support during his doctoral studies. We thank N. Seignour for conducting additional experiments.

[1] J. Magnaudet and I. Eames, The motion of high-Reynolds-number bubbles in inhomogeneous flows, Annu. Rev. Fluid Mech. 32, 659 (2000).

[2] J. Hallez and D. Legendre, Interaction between two spherical bubbles rising in a viscous liquid, J. Fluid Mech. 673, 406 (2011).

[3] F. Takemura and J. Magnaudet, The transverse force on clean and contaminated bubbles rising near a vertical wall at moderate Reynolds number, J. Fluid Mech. 495, 235 (2003).

[4] A. W. G. De Vries, A. Biesheuvel, and L. Van Wijngaarden, Notes on the path and wake of a gas bubble rising in pure water, J. Multiphase Flow 28, 1823 (2002).

[5] M. F. Moctezuma, R. Lima-Ochoterena, and R. Zenit, Velocity fluctuations resulting from the interaction of a bubble with a vertical wall, Phys. Fluids 17, 098106 (2005).

[6] B. Figueroa-Espinoza, R. Zenit, and D. Legendre, The effect of confinement on the motion of a single clean bubble, J. Fluid Mech. 616, 419 (2008).

[7] D. Legendre, J. Magnaudet, and G. Mougin, Hydrodynamic interactions between two spherical bubbles rising side by side in a viscous liquid, J. Fluid Mech. 497, 133 (2003).

[8] R. Zenit and D. Legendre, The coefficient of restitution for air bubbles colliding against solid walls in viscous liquids, Phys. Fluids 21, 083306 (2009).

[9] H. K. Tsao and D. L. Koch, Observation of high Reynolds number bubbles interacting with a rigid wall, Phys. Fluids 9, 44 (1997). 


\section{BARBOSA, D. LEGENDRE, AND R. ZENIT}

[10] P. Aussillous and D. Quéré, Bubbles creeping in a viscous liquid along a slightly inclined plane, Europhys. Lett. 59, 370 (2002).

[11] C. Barbosa, The interaction of bubbles with inclined walls, Ph.D. thesis, Universidad Nacional Autonoma de Mexico, 2016.

[12] D. Legendre, R. Zenit, and J. R. Velez-Cordero, On the deformation of gas bubbles in liquids, Phys. Fluids 24, 043303 (2012).

[13] D. W. Moore, The velocity of rise of distorted gas bubbles in a liquid of small viscosity, J. Fluid Mech. 23, 749 (1965).

[14] H. K. Tsao and D. L. Koch, Collisions of slightly deformable, high Reynolds number bubbles with short-range repulsive forces, Phys. Fluids 6, 2591 (1994). 